\title{
Realization of X-ray telescopes-from design to performance
}

\author{
Bernd Aschenbach
}

Received: 23 March 2009 / Accepted: 26 March 2009 / Published online: 22 April 2009 (C) The Author(s) 2009. This article is published with open access at Springerlink.com

\begin{abstract}
For more than 45 years the building of X-ray telescopes for solar and astronomical observations has been practised with significant performance improvement. The various techniques applied are reviewed emphazising the impact of proper mirror material choice, grinding and polishing improvements and the role of metrology.
\end{abstract}

Keywords Astronomy $\cdot \mathrm{X}$-ray telescopes $\cdot$ Imaging $\cdot$ Grazing incidence

\section{Introduction}

This symposium celebrates the 400th anniversary of the invention of the optical telescope. X-ray telescopes are much younger, not even 60 years of age. Riccardo Giacconi, the "father" of X-ray astronomy and the astronomically used X-ray telescope, has familiarized us with the early beginnings and the scientific success of both X-ray astronomy in general and X-ray telescopes in particular, emphasizing the history in the United States of America. In the late 1960's, early 1970's experimental studies on developing X-ray telescopes were initiated also in several countries in Europe, including the Netherlands, the United Kingdom, Germany and the former Tchechoslovakia, and Italy and Denmark later on. In my talk I will concentrate on the physical principles of X-ray telescopes, the technological and technical challenges and the

B. Aschenbach $(\varangle)$

Max-Planck-Institut für extraterrestrische Physik, Giessenbachstrasse, 85741 Garching, Germany

e-mail: bra@mpe.mpg.de 
performance of the various approaches, which of up to today have resulted in more than ten space-borne missions with $\mathrm{X}$-ray telescopes for astronomical observations.

\section{Grazing-incidence telescopes}

\subsection{The physics of grazing-incidence reflection}

One way to focus and image sources of light is by using reflecting curved surfaces. The interaction of light with matter can be described by the complex index of refraction which describes the change of the properties of the incident electromagnetic wave when crossing the boundary between the two materials involved. The index $n$ reads:

$$
\mathrm{n}=1-\delta-\mathrm{i} \cdot \beta
$$

$\delta$ describes the phase change and $\beta$ accounts for the absorption. The reflection coefficients for $p$ and $s$ polarization are given by the Fresnel equations:

$$
\begin{gathered}
r_{p}=\left(\frac{E_{r}}{E_{i}}\right)_{p}=\frac{n^{2} \sin \alpha-\sqrt{\left(n^{2}-\cos ^{2} \alpha\right)}}{n^{2} \sin \alpha+\sqrt{\left(n^{2}-\cos ^{2} \alpha\right)}} \\
r_{s}=\left(\frac{E_{r}}{E_{i}}\right)_{s}=\frac{\sin \alpha-\sqrt{\left(n^{2}-\cos ^{2} \alpha\right)}}{\sin \alpha+\sqrt{\left(n^{2}-\cos ^{2} \alpha\right)}}
\end{gathered}
$$

$E_{\mathrm{r}} / \mathrm{E}_{\mathrm{i}}$ denotes the ratio of the amplitudes of the reflected and incident electric fields and $\alpha$ is the grazing angle of incidence as measured from the interface plane. For normal incidence, which is the standard in optical telescopes, $\alpha \approx 90^{\circ}$. This approach is, generally speaking, correct as long as the assumptions for applying the Fresnel equations are fulfilled. The reflected intensity or reflectivity is then $\mathrm{R}_{\mathrm{p}}=\mathrm{r}_{\mathrm{p}} \times r_{\mathrm{p}}^{*}$ and $\mathrm{R}_{\mathrm{s}}=\mathrm{r}_{\mathrm{s}} \times r_{\mathrm{s}}^{*}$, where the asterisk denotes the conjugate complex value.

The components of the index of refraction for a vacuum matter transition are often called the optical constants of the material. In the optical wavelength range, for instance, the real part of the index of refraction is greater than one, but with decreasing wavelength its becomes less than one, which changes the interaction of light with matter dramatically. The reflectivity of the surface at normal incidence decreases rapidly and the mirrors lose efficiency starting in the UV wavelength band. However, if one applies Snell's law to the incident and refracted light, it turns out that the refraction angle measured from the surface normal is greater than $90^{\circ}$ for $\mathrm{n}_{\mathrm{r}}=1-\delta<1$, or that total external reflection occurs for grazing-incidence angles $\alpha \leq \alpha_{\mathrm{t}}$ :

$$
\cos \alpha_{\mathrm{t}}=1-\delta
$$


or for $\delta \ll 1$ :

$$
\alpha_{\mathrm{t}}=\sqrt{2 \delta}
$$

For actual applications a trade-off is to be made in terms of the effective collecting area between the design of a normal-incidence telescope and that of a grazing-incidence telecope. The effective collecting area is the product of the wavelength-dependent reflectivity times the geometric area of the primary mirror projected on the front aperture. Depending on the number of reflecting optical elements involved, grazing-incidence telescopes tend to be more efficient for wavelengths shorter than about $30 \mathrm{~nm}$. Furthermore the reflectivity at normal incidence drops so rapidly with decreasing wavelength that for observations at wavelengths shorter than about $15 \mathrm{~nm}$ grazing incidence is the only choice. This limit can be extended somewhat to even shorter wavelengths by the use of multi-layer coatings of the mirror but only over a fairly restricted wavelength band. Multi-layer coatings of several hundreds of bi-layers, each a couple of Ångstro̊m thick, can also be applied to grazing-incidence mirrors, thereby extending the photon energy range to about $100 \mathrm{keV}$.

The index of refraction or the optical constants can be computed from anomalous dispersion theory. For wavelengths $\lambda$ or photon energies sufficiently off-set from any electron binding energy a coarse estimate of $\delta$ can be made:

$$
\delta=\frac{\mathrm{r}_{\mathrm{e}}}{2 \pi} \frac{\mathrm{N}_{0} \rho}{\mathrm{A}} \mathrm{Z} \lambda^{2}
$$

where $\mathrm{N}_{0}$ is Avogadro's number, $\mathrm{r}_{\mathrm{e}}$ is the classical electron radius, $\mathrm{Z}$ and $\mathrm{A}$ are the atomic number and weight, respectively, and $\rho$ is the mass density. For heavy elements for which $\mathrm{Z} / \mathrm{A} \approx 0.5$, the incidence angle of total reflection for $\delta \ll 1$ can be estimated to:

$$
\alpha_{\mathrm{t}}=5.6 \lambda \sqrt{\rho}
$$

with $\alpha_{\mathrm{t}}$ in arcmin, $\lambda$ in $\AA$ and $\rho$ in $\mathrm{g} / \mathrm{cm}^{3}$. For X-rays, with $\lambda$ of a few $\AA$, $\alpha_{\mathrm{t}}$ is about one degree. Equation (7) suggests the most dense materials as reflective coatings like gold, platinum or iridium, which all have been used for $\mathrm{X}$-ray space telescope mirrors. However, these materials show a pronounced reduction of reflectivity at energies between $2 \mathrm{keV}$ and $4 \mathrm{keV}$ because of the presence of M-shell absorption, so that nickel, for instance, despite its lower density has sometimes been preferred, in particular, for observations below $4 \mathrm{keV}$.

The optical constants are related to the atomic scattering factors, the most up-to-date tables of which have been compiled by the Center for X-ray Optics (http://henke.lbl.gov/optical_constants/, [1]). These tables cover the energy range from $50 \mathrm{eV}$ to $30 \mathrm{keV}$ for the elements with $\mathrm{Z}=1-92$, and are a very useful data basis for designing grazing-incidence optics. 


\subsection{Grazing-incidence telescope configurations}

At grazing incidence, imaging of an extended source or imaging over some extended field requires at least two reflections, i.e. two reflecting surfaces. Single mirrors like grazing-incidence parabolas suffer from strong coma, preventing true imaging. However, such a mirror can still focus, and parabolas have been used as 'light buckets'.

There are three different configurations of two-mirror systems, which are the Wolter type systems, the Kirkpatrick-Baez type systems, and the focusing collimator or 'lobster-eye' systems.

\subsubsection{Wolter telescopes}

In 1952 Hans Wolter suggested three different types of imaging telescopes for grazing incidence, which have become known as Wolter telescopes of type I, type II and type III [2]. The surfaces used encompass a paraboloid, a hyperboloid and an ellipsoid. Type I and type II make use of a paraboloid and a hyperboloid, type III combines a paraboloidal and an ellipsoidal mirror. In each case the two mirrors involved are arranged in a coaxial and confocal manner. The main difference between the three types is the ratio of focal length to total system length, i.e. the minimum physical length of the telescope.

The focal length of a type I system (Fig. 1) is practically given by the distance from the paraboloid/hyperboloid intersection plane (Knickfläche) to the system focus. Therefore the physical telescope length always exceeds the focal length by the length of the paraboloid. This system has been mostly used in space observations because of its compactness, simple configuration as far as the interface to the mounting structure is concerned, and because it provides free space to easily add further telescopes inside and outside. These telescopes with multiple components are called nested systems. They increase the collecting area substantially.

Single type I systems have been used for solar X-ray observations whereas for astronomical EUV and X-ray observations, for which collecting area
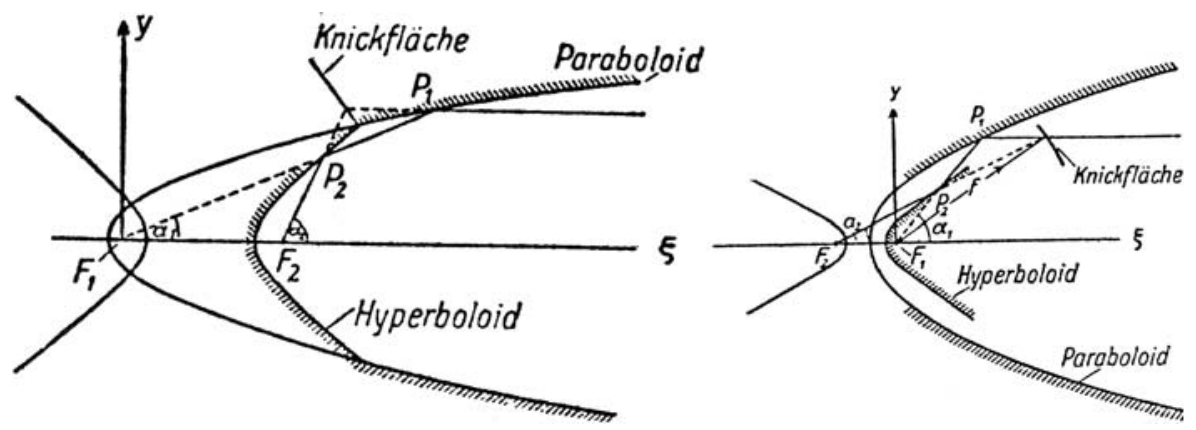

Fig. 1 Schematic of the Wolter telescope type I (left) and type II (right) [2] 
is of utmost importance, nested systems have been used (the EINSTEIN observatory [3] and [4], EXOSAT [5], ROSAT [6], ASCA [7] \& [8] and Suzaku [9], the Chandra [10] and [11] and XMM-Newton [12] observatories, as well as the JET-X telescopes of the SWIFT mission [13] and [14]). For instance, each of the three X-ray telescopes on board of XMM-Newton [12] accommodates 58 nested paraboloid-hyperboloid Wolter type I mirror shell pairs.

The Wolter type II system (Fig. 1) is a true telescopic system, for which the focal length can be much longer than the physical length of the telescope. These systems are useful for feeding spectrometers which require large dispersion.

The f-number is an important number for optical telescopes when imaging extended objects. The lower the f-number is the higher is the image brightness. Likewise f-numbers may also be defined for X-ray telescopes which can be computed using (1)-(7). It turns out that the f-number is inversely proportional to the angle of total reflection which in turn decreases linearly with increasing photon energy. Therefore telescopes optimized for the low-energy regime $(<2$ $\mathrm{keV}$ ) are pretty fast and should make use of the Wolter type I design. The minimum effective f-number of the ROSAT telescope was 9. Telescopes for efficient observations of high-energy photons of up to $10 \mathrm{keV}$ necessarily have much larger f-numbers (around 75 for XMM-Newton or 40 for Chandra), depending on how much emphasis is given to high energies. Type II's should be used if a very long focal length is required compared with the telescope length, because Wolter's "Knickfläche" (c.f. Fig. 1) can easily be positioned far in front of the entrance plane of the primary mirror. Even in the very soft $\mathrm{X}$-ray domain f-numbers of less than 50 (e.g., the $C D S$ telescope of the $S O H O$ solar observatory) cannot be obtained.

Because of the intimate interdependence between f-number, grazing angle, telescope diameter and focal length, large diameter telescopes working at high energies can be constructed only with appropriate long focal distances, and because folding of the X-ray beam is unacceptable because of significant reflection losses, the distance between mirror module and focal plane becomes substantial. This created the idea of space formation flying of two spacecraft, one carrying the telescope and the other one far behind housing the focal plane instrumentation. If the separation is not too large an expandable optical bench might bridge the distance.

Wolter-type systems are free of spherical aberration, but still suffer from coma aberration, astigmatism and field curvature. In a second paper Wolter presented the equations for grazing-incidence telescopes which exactly obey the Abbe sine condition, eliminating coma completely. This is achieved by very small corrections (sub- $\mu \mathrm{m}$ to one $\mu \mathrm{m}$ ) of the axial mirror profile from its nominal second-order shape. The exact surface shape has been derived by Wolter by extending the solutions to grazing incidence which Karl Schwarzschild had already obtained for normal incidence in 1905 [15]. Therefore these systems are named Wolter-Schwarzschild telescopes [16]. They surpass the Wolter systems in off-axis imaging performance if used at longer wavelengths, i.e. in the EUV and the soft X-ray band. Wolter-Schwarzschild type I telescopes 
were flown on the EUV-Explorer [17] and [18] and the ROSAT-WFC [19]. A Wolter-Schwarzschild type II system was feeding the spectroscopic telescope of the EUV-Explorer, and the CDS telescope on board of the solar SOHO mission [20] is of Wolter-Schwarzschild type II.

The maximum degree of nesting, and therefore the highest throughput relative to the entrance aperture area, is achieved with mirrors as thin as possible. Hundreds of thin foils or sheets representing the mirrors make up the telescopes used in the $A S C A$ and Suzaku missions. The parabolic/hyperbolic shape of the Wolter type I mirrors is approximated by straight cones. The perfect image of an on-axis point source is lost but the imaging capability is preserved. Cone approximation of the Wolter type I configuration has also been used for the BeppoSax X-ray telescopes [21] \& [22] (Figs. 2, 3, 4, 5, and 6).

\subsubsection{Kirkpatrick-Baez telescopes}

The first two-dimensional X-ray image ever obtained with grazing-incidence reflection was taken in the laboratory by Kirkpatrick and Baez [23]. The incident rays are focused to a line image by a parabolic mirror. On their path to the line focus the rays are reflected by a second parabolic mirror to the

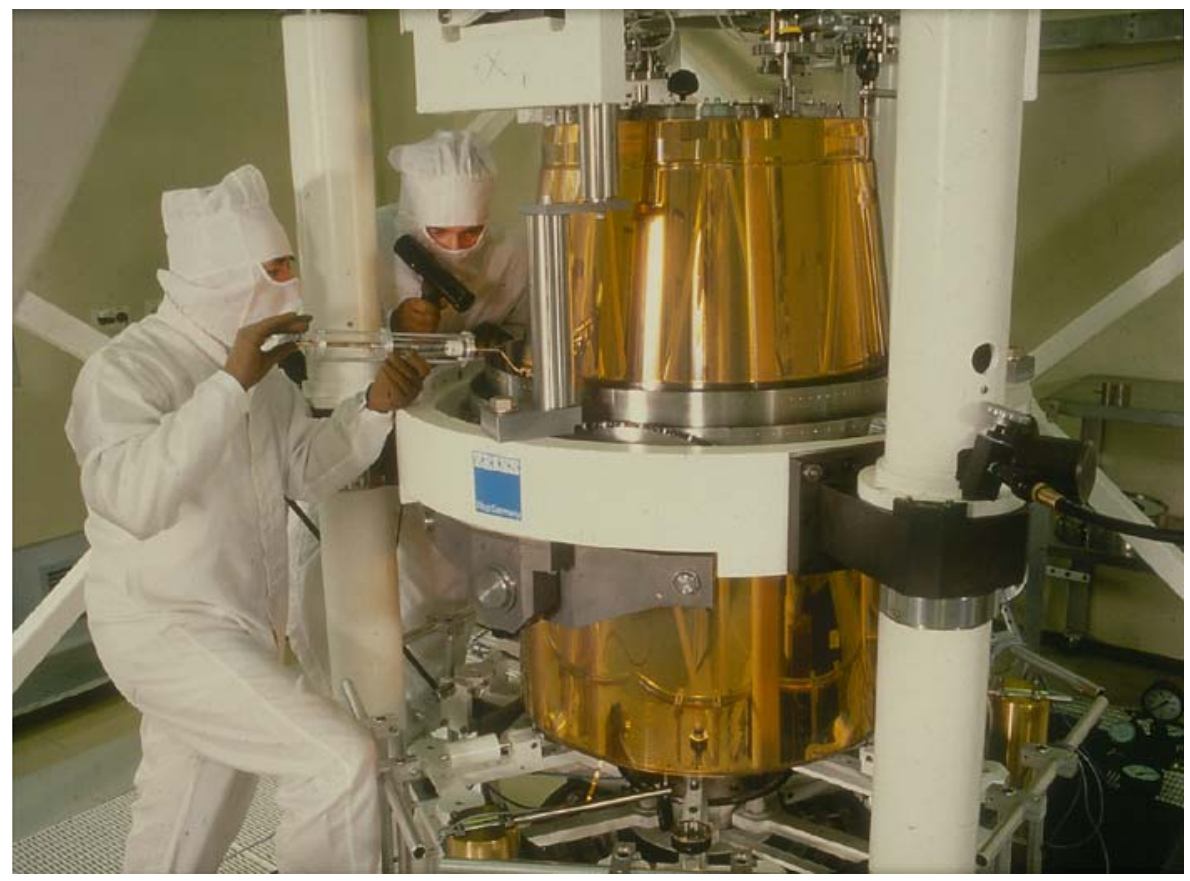

Fig. 2 Bonding of one of the ROSAT hyperboloid mirrors to the central bulkhead of the telescope which eventually contains 8 separate, Zerodur-made paraboloid and hyperboloid mirrors 
Fig. 3 Integration of the secondary mirror of the $C D S$ telescope, which is of

Wolter-Schwarzschild type II. Both the primary (bottom mirror) and the secondary (top mirror) as well as the carrying structure are made solely out of Zerodur.

Grinding and polishing of the mirrors was particularly challenging because of the extreme asphericity of the surfaces. The telescope finally has an angular resolution of less than 2.5 arcsec HEW. This telescope has an aperture diameter of $275 \mathrm{~mm}$, and despite the long focal length of $2578 \mathrm{~mm}$ the separation between front aperture and focal plane ist just $800 \mathrm{~mm}$

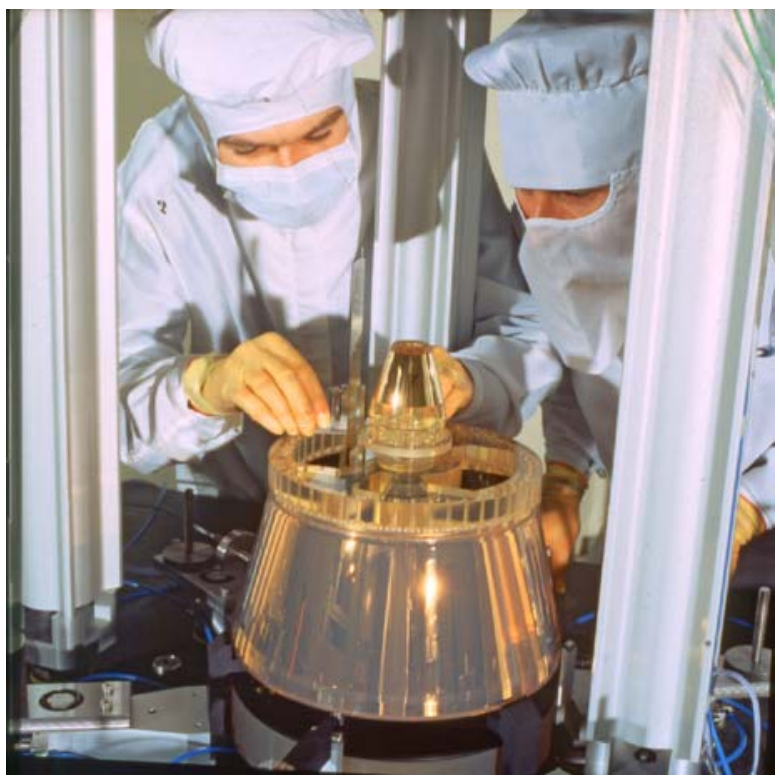

point-like focus for rays parallel to the centre lines of the parabolas. The surface planes of the two mirrors are oriented at $90^{\circ}$ to each other. In order to increase the collecting area (the frontal area) a stack of parabolas of translation can be constructed. However, in contrast to the single double-plate system, the image of a point-like source starts to become increasingly extended in size as the number of plates involved increases. Wolter type I telescopes bend the incident ray direction two times in the same plane, whereas the two bendings in Kirkpatrick-Baez systems occur in two orthogonal planes, which for the same incidence angle on the primary mirror requires a longer telescope.

A Kirkpatrick-Baez telescope has never been flown on a satellite mission, but a modification using flat plates instead of parabolas, still providing twodimensional imaging, has successfully operated on sounding rocket flights delivering positive measurements of ordinary stars and clusters of galaxies [24].

\subsubsection{Focusing collimator or 'lobster-eye' telescopes}

The Wolter and the Kirkpatrick-Baez systems have in common a relatively narrow field of view which is practically limited to the grazing angle employed on the individual mirrors. Imaging systems of substantially larger field of view but at systematically reduced on-axis angular resolution have been proposed by Schmidt [25] and by Angel [26]. Such systems would be ideal for a wide field imaging monitor.

The principal layout of Schmidt's concept makes use of two stacks of plane mirrors, which are arranged in an upper and a lower stack and oriented orthogonally to each other. The mirrors within each stack are arranged in 


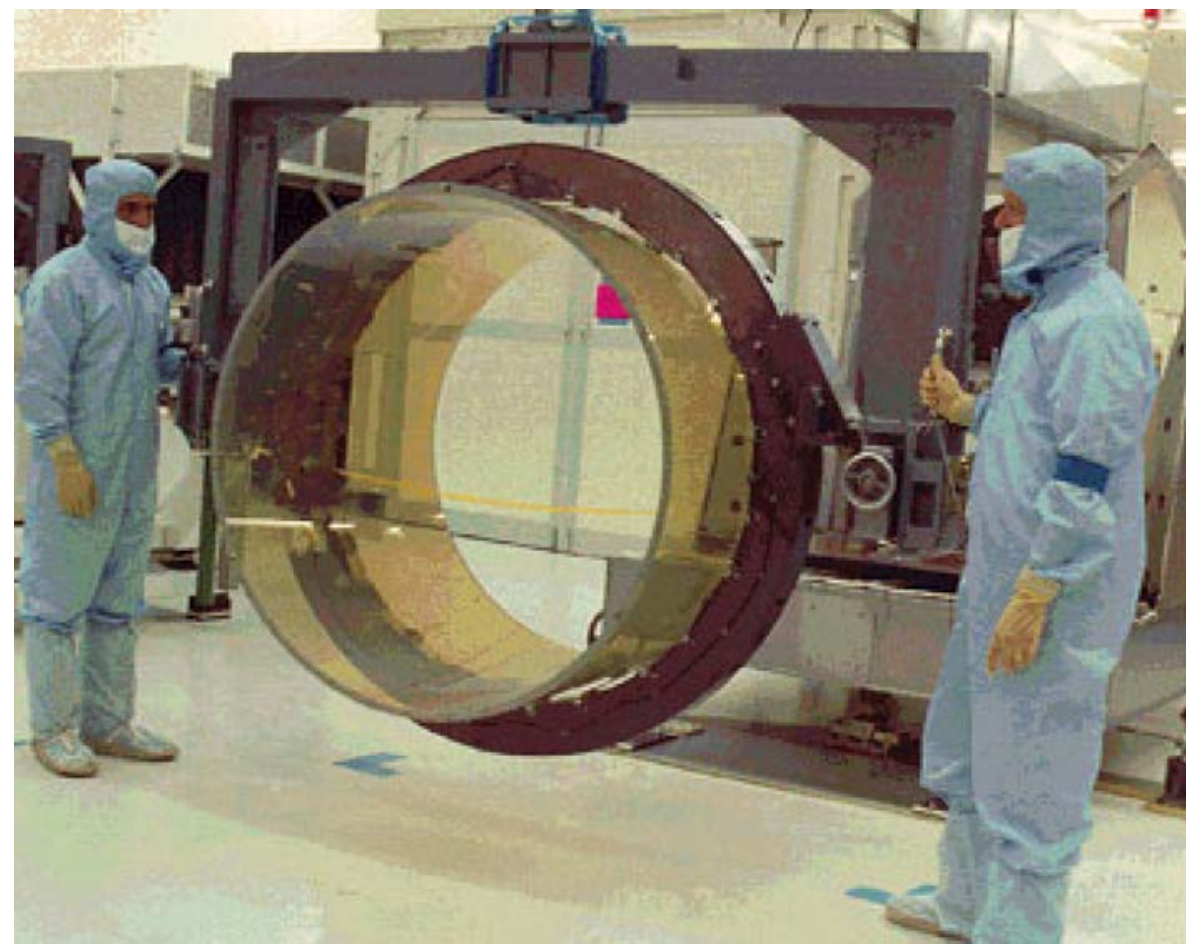

Fig. 4 Riccardo Giacconi's “dream" mirror, the $1 \mathrm{~m}$ long and $1.2 \mathrm{~m}$ wide paraboloidal Zerodur mirror which makes up the biggest mirror of the 0.5 arcsec Chandra telescope. According to him this diameter of an X-ray mirror is of the size he has always been opting for since the beginning of telescopic X-ray astronomy. The biggest mirror of the earlier Einstein telescope had a diameter almost exactly half of it (Image credit: NASA/CXC/SAO)

such a way that their center lines inscribe a cylinder, where the two cylinders associated with the stack are at right angles to each other and the crossing of their center lines is at the origin of the coordinate system. A focus is formed half way between the mirrors and the origin of the coordinate system. Both sides of a mirror blade, i.e. the front and the back surface, are X-ray reflecting. The focusing is not perfect because of the finite height of the mirror blades. With such a device a full hemisphere of the sky could be observed simultaneously.

A variation of this design, which provides two-dimensional imaging, has been presented by Angel [26] (see also [27] and references therein). The device is composed of many small square-sided tubes with reflecting surfaces. The tubes are based on and distributed over the surface of a sphere. The axis of each tube follows a radius vector of the sphere. After a ray has been reflected twice within one tube but from adjacent walls a two-dimensional image is formed. The focal surface is a sphere with a radius which is half of that of the sphere carrying the tubes. This type of grazing-incidence optic is actually 
Fig. 5 View to the rear of one of the three

XMM-Newton Wolter I telecopes. There are 58 nested mirror shells produced in nickel via galvanic replication including gold plating.

Parabola and hyperbola come in one piece with a thickness between $0.5 \mathrm{~mm}$ and $1.2 \mathrm{~mm}$, spanning a diameter range from $35 \mathrm{~cm}$ to $70 \mathrm{~cm}$ with a length of $60 \mathrm{~cm}$

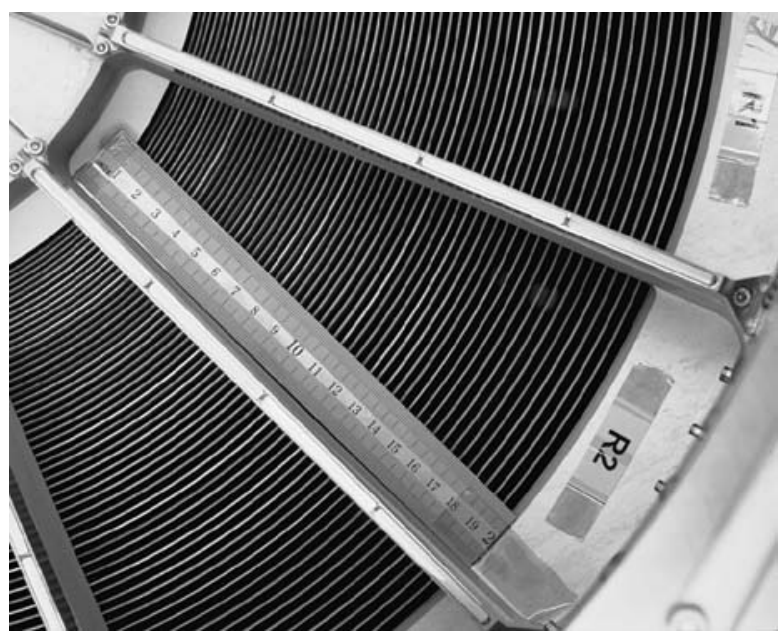

realized in the reflective eyes of lobsters and shrimps, giving the name to this particular type of X-ray telescope. The optical principle is very similar to Schmidt's focusing collimator when shifting and merging the upper and lower mirror stacks of Schmidt's device into one section forming square-sided tubes.

Both in the Schmidt and the Angel design there are rays passing through the optics with only one reflection or none at all. They appear as a diffuse or lineshaped background of non-negligible brightness. In itself the imaging is not perfect, and, ultimately, the angular resolution of such a device is limited by the width of a single tube as seen by the detector, and at arcsecond resolution the diffraction imposed by the tube width has to be taking into account, favouring such a system for observing hard X-rays. Such a telescope would have great potential for continuous X-ray monitoring of large fields of the sky.

Fig. 6 One of the four X-ray telescope modules on board of the Suzaku satellite. The module houses a total of 175 shells or 1400 reflectors. The mirror shells are extremely thin $(0.152 \mathrm{~mm})$ aluminum foils, each of which is about $12 \mathrm{~cm}$ long [9]. This technique has been pioneered by Peter Serlemitsos in the late 1980's [7], and was also used for the fabrication of the $A S C A$ telescopes [8]

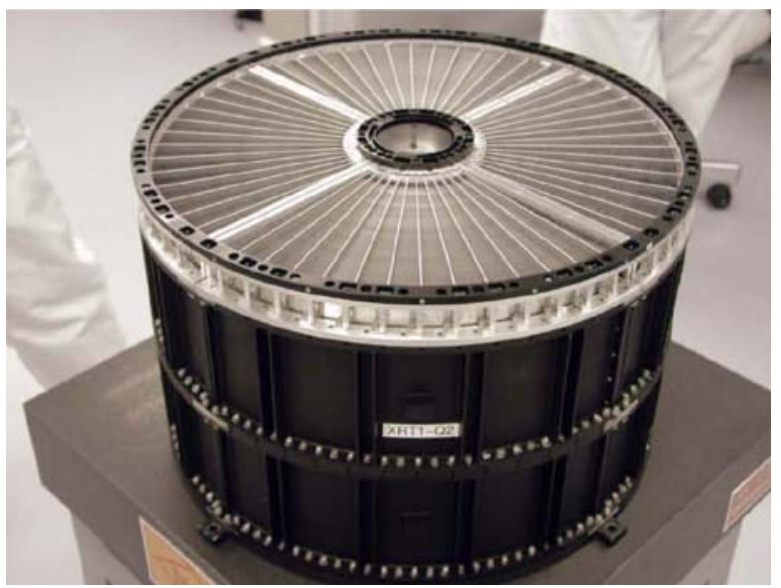


Prototypes have been constructed and built in the Czech Republic by the group of Hudec et al. [28].

\section{Design considerations}

\subsection{Parameters}

The choice of a particular type of grazing-incidence telescope depends on the scientific objectives. First of all, the highest photon energy has to be considered, which according to (7) defines the optimum grazing angle. Such energies are found, for instance, in the astrophysically important Fe K-lines between 6.4 and $7 \mathrm{keV}$. The $X M M$-Newton telescope was designed to optimally cover this region and to provide an acceptable area at $2 \mathrm{keV}$.

Surveying telescopes should have a low f-number, which is equivalent to maximizing the product of the effective collecting area times the field of view. This requires a grazing angle as large as possible but compatible with the average photon energy within the band to be observed. The ROSAT telescope was designed along these lines.

Furthermore, such a survey telescope should have the best possible angular resolution over a field of view as wide as possible. Wolter type I telescopes show strong field curvature, which means that the angular resolution increases rapidly with growing field angle (off-axis angle) [29]. So-called polynomial telescopes tend to flatten the field [30] and [31]. They maintain the Wolter configuration but the second-order surface shape is replaced by higher-order polynomials at the expense of degrading the on-axis resolution. Alternatively, for nested systems, the primary mirrors of a Wolter telescope should be kept as short as possible without compromising the free entrance aperture.

\subsection{Effective collecting area}

These trade-offs are usually done by detailed ray-tracing taking into account the reflectivity of the coating, which usually changes substantially with energy. Figure 7 shows the effective collecting area of one of the three XMM-Newton telescopes. The widest of the 58 nested Wolter type I mirror pairs has a diameter of $70 \mathrm{~cm}$. The geometric area of the entrance aperture is about $3100 \mathrm{~cm}^{2}$ and the effective area at the lowest energies (c.f. Fig. 7) is about $1850 \mathrm{~cm}^{2}$, equivalent to a throughput of less than $60 \%$. This can be increased by the use of thinner mirrors like foils or thin sheets with which a throughput of more than $80 \%$ has been achieved. The on-axis angular resolution of such foil telescopes, however, is so far limited to about one arcminute.

\subsection{Angular resolution, point spread function, encircled energy function}

Even for a perfect telescope the light from a point-like object located on-axis is not concentrated in an infinitely small focal spot, but is distributed over 
Fig. 7 On-axis effective collecting area of one of the three $X M M$-Newton X-ray telescopes, as designed. All 58 mirror pairs have been coated with gold, which causes the energy dependence of the area. The jumps and wiggles are due to the binding energies of the N-, M- and L-shell electrons

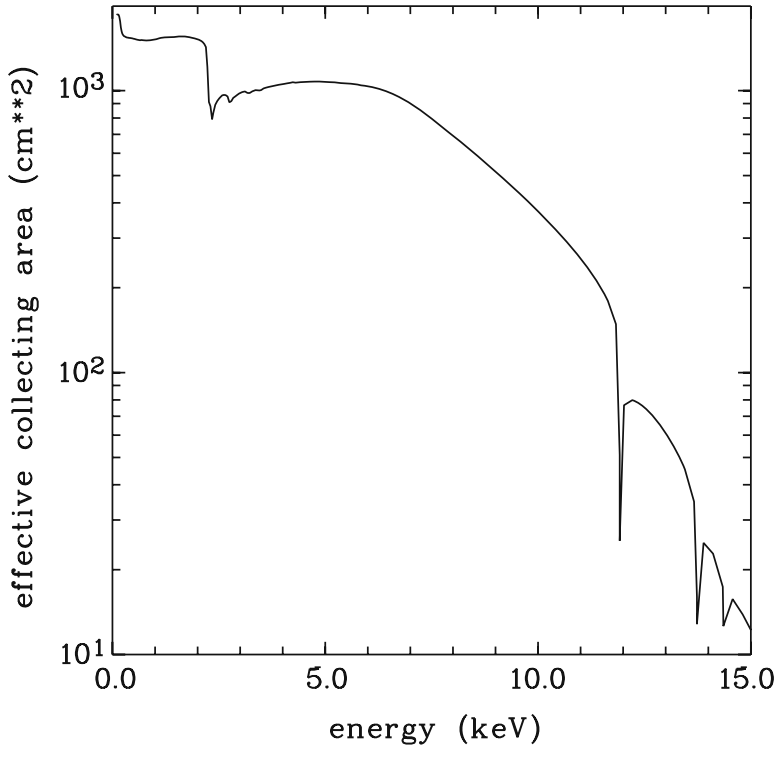

an extended image, the surface brightness of which is described by the point spread function (PSF). The encircled energy function (EEF) corresponds to the radial integration of the PSF, and describes the relative fraction of effective area within some radius.

At large field angles the image extent and structure are dominated by geometric aberrations inherent in the telescope design. In contrast, the onaxis PSF is determined by differences between the real and perfect shape of the reflecting surfaces, both in circumferential and in axial direction, as well as by alignment and mounting errors. For high-resolution telescopes, the most demanding factor is the control of the surface shape by appropriate metrology, through which a highly precise feedback to computer-controlled grinding and polishing of the relevant surfaces down to residuals of a few Ångstro̊m on either the mirrors or mandrels has become possible. Because of grazing incidence the tightest requirements have to be observed for surface errors along the mirror meridional or axial profiles. They need to be as low as possible over all spatial frequency scales which range from the full mirror length up to the micrometer range. The same holds for circumferential errors.

Low-frequency errors can be considered as geometric slope errors and they can be treated by geometric optics. At high frequencies the errors and their power spectral density distribution can be understood as scattering from randomly rough surfaces [32]. The fraction of scattered X-rays $I_{S} / I_{0}$ is given by:

$$
\mathrm{I}_{\mathrm{s}} / \mathrm{I}_{0}=1-\exp \left[-(4 \pi \sigma \sin \alpha / \lambda)^{2}\right]
$$

The microroughness $\sigma$ is the rms value of the surface height deviations. Microroughness reduces the image contrast, produces a loss of flux out of the 
image core and raises extended wings in the PSF. In the early days of grazingincidence mirrors scattering was a major problem because of the low values for $\sigma$ to be achieved for a reasonable high contrast image. For instance, for $\mathrm{I}_{\mathrm{s}} / \mathrm{I}_{0}=0.1$ at X-ray wavelengths $(\lambda=10 \AA)$ and grazing incidence $\left(\alpha=1^{\circ}\right) \mathrm{a}$ microroughness of $\sigma \leq 9 \AA$ is required. Values like $3 \AA$ have become possible on highly aspherical grazing-incidence mirrors and have been realised on the ROSAT telescope mirrors.

In the early years of making X-ray mirrors scattering was not well understood, and only very extensive X-ray scattering measurements on flat samples showed that (8) was not only applicable to radio and optical wavelengths but also to the X-ray domain (c.f. Fig. 8, [33]). Scalar scattering theory and first order vector perturbation theory in the smooth-surface limit were shown to describe the observed scattering profiles when compared with metrology data. They were obtained with long-range, high-resolution, contactless profilometers, and later, in the late 1980's optical heterodyne interferometers with a vertical resolution capability down to the Ångstro̊m level became available. These sophisticated metrology tools, including extremely precise roundness measurement devices, provided the necessary feedback to the manufacturers for improving grinding and polishing, which now has reached the level of subarcsecond X-ray imaging with mirror areas covering many square meters.

The shape of the PSF core is generated by geometric errors, the total of which accumulates to less than 0.1 arcsecond for the Chandra telescope. Figure 9 shows the on-axis EEFs for the Chandra telescope at various photon energies. Energy-dependent scattering, as expected from (8), appears to be present.

Fig. 8 Comparison of total integrated scatter (TIS) measurements with electromagnetic scattering theory (Equation 8, [33])

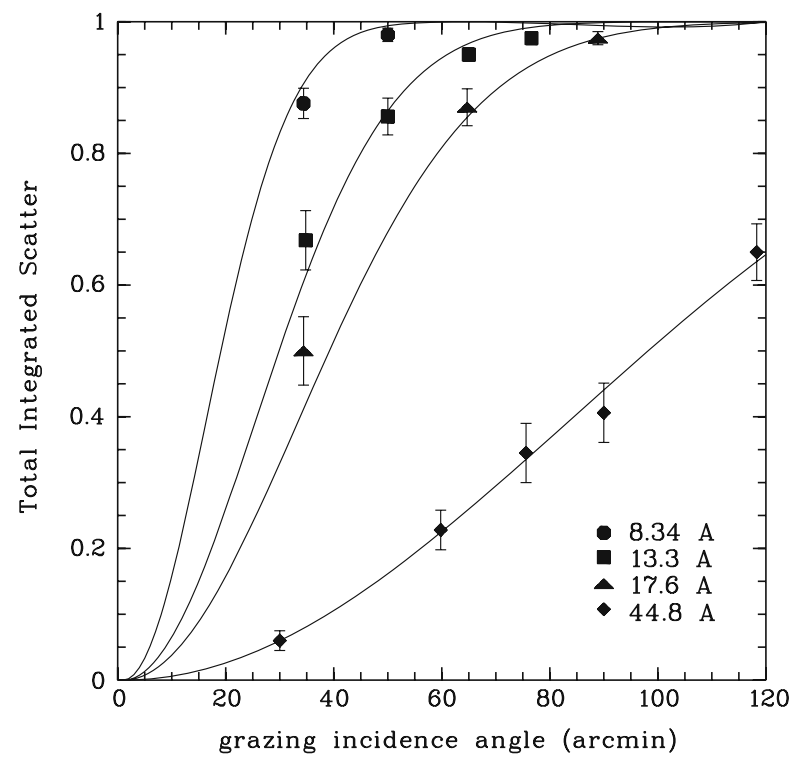


Fig. 9 Encircled energy function of the Chandra telescope for different photon energies [34]

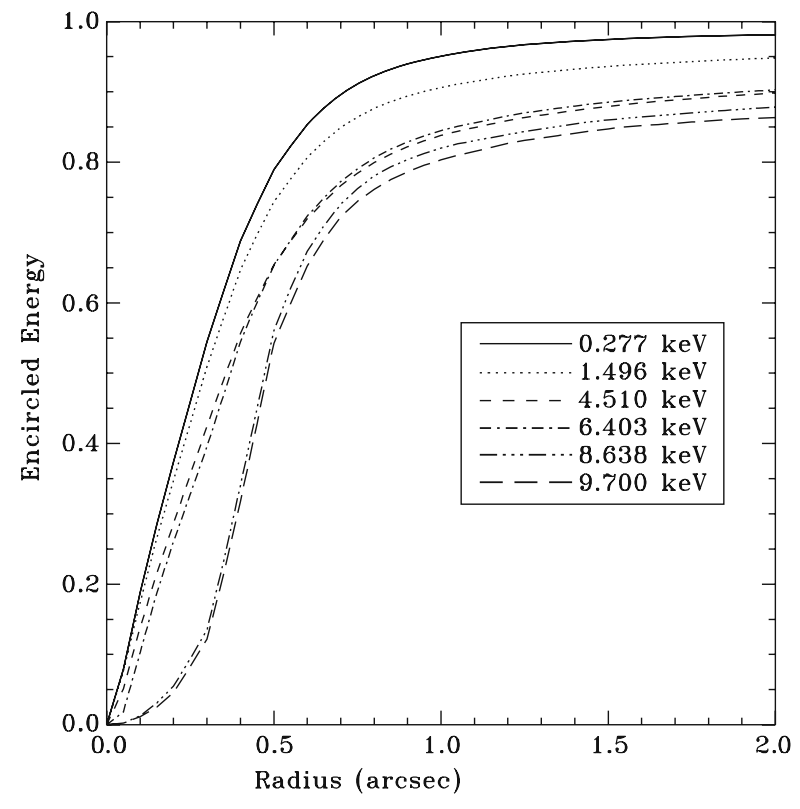

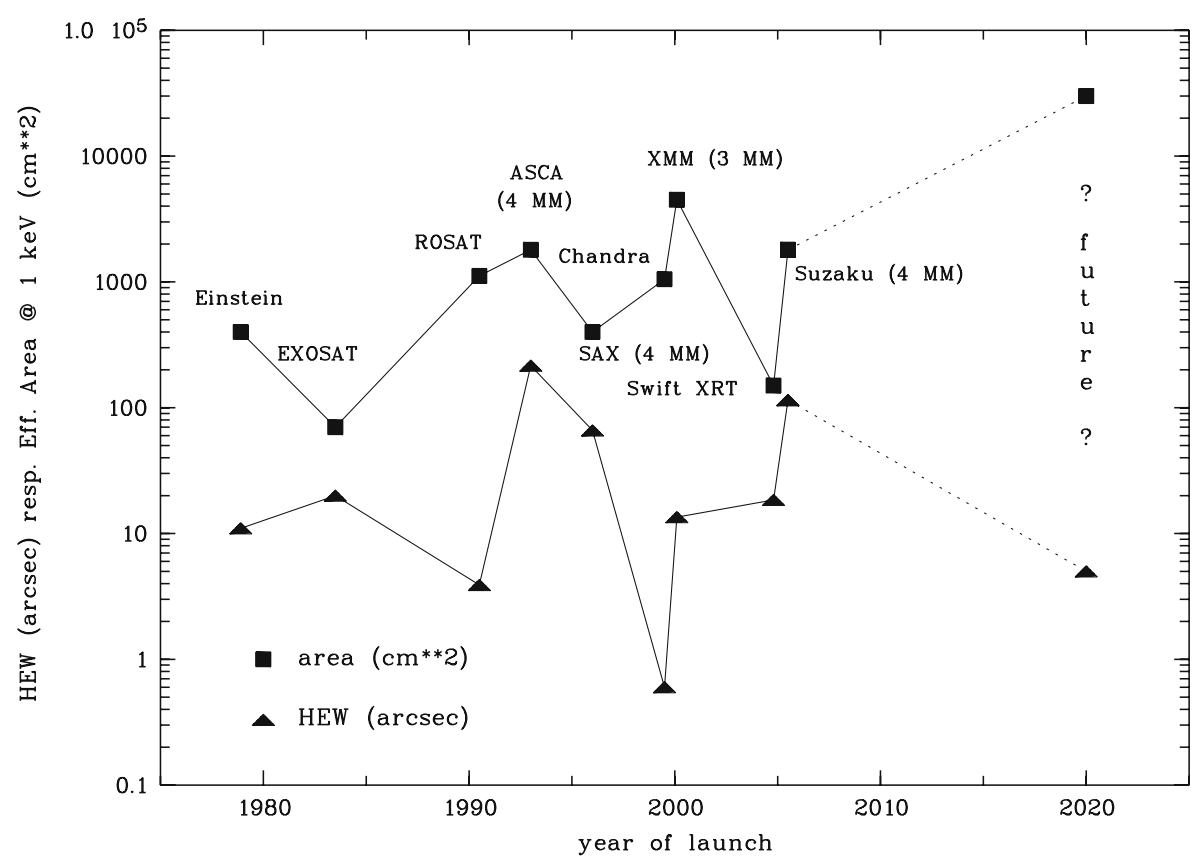

Fig. 10 Performance comparison of various X-ray telescopes, effective collecting area in $\mathrm{cm}^{2}$ (upper data points) and angular resolution in terms of the half energy width (HEW) of the point spread function in arcsec at a photon energy of $1 \mathrm{keV}$ (lower data points). MM stands for mirror modules. Dashed lines are pointing to a future super X-ray telescope with a collecting area of $3 \mathrm{~m}^{2}$ and 5 arcsec resolution currently under study, which would explore the deep Universe. Needless to say that quite a number of other X-ray telescope space missions are being worked on 
A summary of the angular-resolution and effective-collecting-area performance of the telescopes, which have successfully been used on astronomical satellites, is displayed in Fig. 10.

\subsection{Baffling}

Grazing-incidence telescopes have to be equipped with field stops to baffle the focal plane against stray light. Otherwise rays from outside the nominal field of view can pass through the telescope by a single reflection from either the primary or the secondary. There may even be portions of the sky which can be viewed directly from the focal plane detector. Such straylight increases the background light and produces ghost images. A complete suppression of straylight requires field stops in several different planes, which are best in front of the telescope aperture and down in the telescope's mirror section. For tightly nested systems with little if any available space between adjacent mirrors a single baffle system in front of the telescope can be used, which, however, reduces but does not completely eliminate stray light.

Open Access This article is distributed under the terms of the Creative Commons Attribution Noncommercial License which permits any noncommercial use, distribution, and reproduction in any medium, provided the original author(s) and source are credited.

\section{References}

1. Henke, B.L., Gullikson, E.M., Davis, J.C.: X-ray nteractions: photoabsorption, scattering, transmission, and reflection at $\mathrm{E}=50-30,000 \mathrm{cV}, \mathrm{Z}=1-92$. At. Data Nucl. Data Tables 54(2), 181-342 (1993)

2. Wolter, H.: Spiegelsystem streifenden Einfalls als abbildende Optiken für Röntgenstrahlen. Ann. Phys. 10, 94-114 (1952a)

3. Van Speybroeck, L.P.: Einstein observatory /HEAO-B/ mirror design and performance. SPIE Proc. 184, 2-10 (1979)

4. Giacconi, R., Branduardi, G., Briel, U. et al.: The Einstein /HEAO 2/ x-ray observatory. ApJ 230, 540-550 (1979)

5. de Korte, P.A.J., Giralt, R., Coste, J.N. et al.: EXOSAT x-ray imaging optics. Appl. Opt. 20, 1080-1088 (1981)

6. Aschenbach, B.: Design, construction, and performance of the ROSAT high-resolution x-ray mirror assembly. Appl. Opt. 27, 1404-1413 (1988)

7. Serlemitsos, P.J.: Conical foil x-ray mirrors: performance and projections. Appl. Opt. 27, 14471452 (1988)

8. Serlemitsos, P.J., Jahoda, L., Soong, Y. et al.: The x-ray telescope on board ASCA. Pub. Astron. Soc. Jap. 47, 105-114 (1995)

9. Serlemitsos, P.J., Soong, Y., Chan, K.-W. et al.: The x-ray telescope onboard Suzaku. Pub. Astron. Soc. Jap. 59, 9-21 (2007)

10. Van Speybroeck, L.P.: Grazing incidence optics for the U.S. high-resolution x-ray astronomy program. Appl. Opt. 27, 1398-1403 (1988)

11. Van Speybroeck, L.P., Jerius, D., Edgar, R.J. et al.: Performance expectation versus reality. SPIE Proc. 3113, 89-104 (1997)

12. Aschenbach, B., Briel, U.G., Haberl, F. et al.: Imaging performance of the XMM-Newton X-ray telescope. SPIE Proc. 4012, 731-739 (2000)

13. Citterio, O., Campano, S., Conconi, P. et al.: Characteristics of the flight model optics for the JET-X telescope onboard the Spectrum-X-Gamma satellite. SPIE Proc. 2805, 56-65 (1996) 
14. Burrows, D.N., Hill, J.E., Nousek, J.A. et al.: The Swift x-ray telescope. Space Sci. Rev. 120, 165-195 (2005)

15. Schwarzschild, K.: Untersuchungen zur geometrischen Optik. Abh. Wiss. Göttingen Bd. IV Nr. 2 (1905)

16. Wolter, H.: Verallgemeinerte scawarzschildsche spiegelsysteme streifender reflextion als optiken für röntgenstrahlen. Ann. der Phys. 10, 286-295 (1952b)

17. Bowyer, S., Green, J.: Design of the extreme ultraviolet explorer long-wavelength grazing incidence telescope optics. Appl. Opt. 27, 1414-1422 (1988)

18. Bowyer, S., Malina, R.F.: The extreme ultraviolet explorer mission. Adv. Space Res. 11, 205215 (1991)

19. Willingale, R.: ROSAT wide field camera mirrors. Appl. Opt. 27, 1423-1429 (1988)

20. Harrison, R.A., Sawyer, E.C., Carter, M.K. et al.: The coronal diagnostic spectrometer for the solar and heliospheric observatory. Sol. Phys. 162, 233-290 (1995)

21. Citterio, O., Conti, G., Mattaini, E., Santambrogio, E., Sacco, B.: Optics for x-ray concentrators on board of the astronomy satellite SAX. SPIE Proc. 597, 102-110 (1986)

22. Citterio, O., Bonelli, G., Conti, G. et al.: Optics for the x-ray imaging concentrators aboard the x-ray astronomy satellite SAX. Appl. Opt. 27, 1470-1475 (1988)

23. Kirkpatrick, P., Baez, A.V.: Formation of optical images by x-rays. J. Opt. Soc. Am. 38, 766774 (1948)

24. Gorenstein, P., Gursky, H., Harnden, F.R., Jr., DeCaprio, A., Bjorkholm, P.: Large area soft $\mathrm{x}$-ray imaging system for cosmic $\mathrm{x}$-ray studies from rockets. IEEE Trans. Nucl. Sci. NS-22, 616-619 (1975)

25. Schmidt, W.K.H.: A proposed $\mathrm{x}$-ray focusing device with wide field of view for use in $\mathrm{x}$-ray astronomy. Nucl. Instrum. Methods 127, 285-292 (1975)

26. Angel, J.R.P.: Lobster eyes as x-ray telescopes. ApJ 233, 364-373 (1979)

27. Priedhorsky, W.C., Peele, A.G., Nugent, K.A.: An x-ray all-sky monitor with extraordinary sensitivity. Mon. Not. R. Astron. Soc. 279, $733-750$ (1996)

28. Hudec, R., Inneman, A., Pína, L., Hudcová, V.: Lobster eye: new approach to monitor GRBs in x-rays. AIP Conf. Proc. 662, 494-496 (2003)

29. VanSpeybroeck, L.P., Chase, R.C.: Design parameters of paraboloid-hyperboloid telescopes for x-ray astronomy. Appl. Opt. 11, 440-445 (1972)

30. Burrows, C.J.,Burg, R., Giacconi, R.: Optimal grazing incidence optics and its application to wide-field x-ray imaging. ApJ 392, 760-765 (1992)

31. Conconi, P., Campana, S.: Optimization of grazing incidence mirrors and its application to surveying x-ray telescope. Astron. astrophys. 372, 1088-1094 (2001)

32. Aschenbach, B.: Boundary between geometric and wave optical treatment of $\mathrm{x}$-ray mirrors. SPIE Proc. 5900, 59000D-1-59000D-7 (2005)

33. Lenzen, R.: Imaging properties of a Wolter-I type x-ray telescope with particular reference to contrast reduction through diff use reflection. PhD thesis, Universität Tübingen, Germany, 123 pp. (1978)

34. Chandra Proposers' Observatory Guide: Rev.6.0, TD 403.00.006, pp. $36-38$ (2003) 